\title{
Développement post-natal du pouvoir de concentration rénal, du système rénine-angiotensine et des hormones cortico-surrénaliennes chez le veau
}

\author{
M Abouzite ${ }^{1}$, MB Aldaker ${ }^{1}$, S Fellat ${ }^{2}$, \\ H Sahibi ${ }^{3}$, Kh Baddouri ${ }^{1 *}$ \\ ${ }^{I}$ Laboratoire de physiologie animale, département de biologie, faculté des sciences, \\ université Mohammed $\mathrm{V} ;{ }^{2}$ Laboratoire de la ligue cardio-vasculaire, hôpital Ibn-Sina; \\ ${ }^{3}$ Institut agronomique et vétérinaire Hassan II, Rabat, Maroc
}

(Reçu le 17 avril 1996 ; accepté le 14 février 1997)

\begin{abstract}
Summary - Post-natal development of the kidney concentrating ability, the renine-angiotensin system and the cortico-surrenal hormones in the calf. Post-natal development of plasma and urine osmolality, as well as plasma renin activity, aldosteron and cortisol levels were studied in calves between 1 and 9 months of age. The ratio of urine to plasma osmolality rose from 0.8 at an early post-natal age to 3.8 at the end of the study period. Plasma renin activity, plasma aldosteron and cortisol levels did not show any change during the post-natal development period. The plasma concentrations of these hormones were in the same range as is found in the adult.
\end{abstract}

osmolality / development / post-natal / renin / aldosteron / cortisol / calf

Résumé - L'osmolalité plasmatique et urinaire, ainsi que l'activité rénine plasmatique, l'aldostéronémie et la cortisolémie ont été mesurées chez le veau âgé d' 1 à 9 mois. Le rapport osmolaire urine/plasma est de 0,8 chez les veaux âgés d' 1 mois, alors qu'il passe à 3,8 chez les veaux de 9 mois. L'activité rénine plasmatique, l'aldostéronémie et la cortisolémie ne varient pas significativement au cours du développement post-natal, ces hormones ayant les mêmes concentrations que celles de l'adulte.

osmolalité / développement / post-natal / rénine / aldostérone / cortisol / veau

* Correspondance et tirés à part

Fax: (212) 7775461 


\section{INTRODUCTION}

La production d'une urine à pression osmotique élevée chez les mammifères résulte de l'organisation anatomique des néphrons dans le rein, en particulier l'arrangement parallèle des branches descendantes et ascendantes des anses de Henlé, des canaux collecteurs ainsi que des anses vasculaires. La circulation par contre-courant qui en découle permet la création et le maintien d'un gradient axial de pression osmotique dans la médullaire rénale. La concentration osmolaire de l'urine finale dépend aussi de la présence de l'hormone antidiurétique dans le sang. De plus, chez beaucoup de mammifères, le degré d'hétérogénéité néphronique et la capacité du rein à concentrer les urines sont intimement liés (Baddouri et al, 1975 ; Valtin, 1977). Or, le développement morpho-fonctionnel qui se poursuit après la naissance se caractérise par l'augmentation du nombre et de la taille des néphrons, par la différenciation morphologique et fonctionnelle des différents segments, ainsi que par le développement de la microvasculature rénale (Horster et Valtin, 1971).

Le présent travail vise à déterminer l'âge des animaux à partir duquel le pouvoir de concentration rénal est maximal. Les concentrations circulantes de l'activité rénine plasmatique (ARP) et des hormones cortico-surrénaliennes ont été évaluées au cours du développement post-natal chez le veau.

\section{MATÉRIELS ET MÉTHODES}

Les expérimentations ont été conduites sur des veaux mâles de race pie-noire, âgés de 1 à 9 mois à raison de trois veaux par tranche d'âge et ayant un poids corporel compris entre $32 \mathrm{~kg}$ et $96 \mathrm{~kg}$.

Ces animaux recevaient durant les 2 premiers mois un aliment d'allaitement (environ 2,5 L par repas) distribué deux fois par jour. À partir du $3^{\mathrm{e}}$ mois, une nourriture à base d'orge, de mélasse, et de l'eau fraîche était continuellement disponible.
Les échantillons sanguins ont été prélevés le matin durant la saison d'été à raison d'un prélèvement par mois et par animal. Ces prélèvements sont effectués avant ceux des urines pour minimiser le plus possible un stress éventuel. Le sang a été prélevé par ponction de la veine jugulaire dans des tubes préalablement refroidis et contenant, selon la nature de la substances biologique à doser, de l'EDTA $(15 \%, \mathrm{pH}=6,5)$ avec un inhibiteur des protéases (PMSF) pour les dosages hormonaux, ou un anticoagulant (anticlot) pour la mesure de la pression osmotique plasmatique. Le sang était centrifugé à $3000 \mathrm{rpm}$ à $4^{\circ} \mathrm{C}$ pendant $15 \mathrm{~min}$, et le plasma était récolté pour les dosages.

Les urines ont été recueillies chez les veaux par friction de l'organe génital et compression de la région abdominale (le $S$ pénien chez le bovin permet très difficilement l'accès à la vessie à partir de l'orifice urinaire ; Baron, 1990).

Les pressions osmotiques plasmatique et urinaire ont été déterminées par la mesure de l'abaissement du point de congélation de ces liquides (osmomètre Roebling).

Le cortisol, l'ARP (activité rénine plasmatique) ont été dosés par méthode radio-immunologique directe dans les échantillons plasmatiques. Le dosage de l'ARP a été réalisé avec le Kit CIS Biointernational Ren-CT2 ; la limite de détection est de $0,15 \mathrm{ng} / \mathrm{d}$ 'angiotensine $\mathrm{I}$. Le coefficient de variation intra et inter-essai est estimé respectivement à $7,2 \%$ et $8,2 \%$.

L'aldostérone a été mesurée à l'aide du Kit CIS Biointernational : la limite de détection est de $15 \mathrm{pg} / \mathrm{mL}$ et le coefficient de variation intra et inter-essai est respectivement de $9,5 \%$ et $11,5 \%$. Le cortisol a été dosé à l'aide du Kit CIS biointernational ; la sensibilité du dosage est de 4,6 $\mathrm{nmol} / \mathrm{L}$

Les valeurs représentent la moyenne arithmétique accompagnée de l'erreur standard sur la moyenne (X士ESM).

La signification des différences observées est appréciée en utilisant le test de Fisher (test PPDS), après analyse de la variance à un seul facteur.

Dans la discussion, seules les différences affirmées avec un risque inférieur à $5 \%(p<0,05)$ sont considérées comme significatives ou probables. 


\section{RÉSULTATS}

\section{Osmolalité plasmatique}

Les données relatives à l'évolution de l'osmolalité plasmatique au cours du développement post-natal chez les veaux sont illustrées dans le tableau I. L'osmolalité plasmatique ne varie pas significativement au cours des 9 premiers mois chez le veau (Anova à un seul facteur, $F=0,664, p=0,715$ ).

\section{Osmolalité urinaire}

L'osmolalité urinaire augmente significativement en fonction de l'âge $(p=0,0001)$. D'une valeur de $242 \pm 42 \mathrm{mosmol} / \mathrm{kg}$ à l'âge d'1 mois, l'osmolalité urinaire s'élève à 491 $\pm 23 \mathrm{mosmol} / \mathrm{kg}$ au $3^{\mathrm{e}}$ mois $(p<0,01)$ et à $828 \pm 42 \mathrm{mosmol} / \mathrm{kg}$ au $4^{\mathrm{e}}$ mois $(p<0,001)$, puis se stabilise (tableau I).

\section{Rapport osmolalité urinaire/ osmolalité plasmatique (U/P)}

L'évolution post-natale du rapport $U / P$ est reportée dans le tableau $I$ où apparaît une augmentation significative en fonction de l'âge (Anova à un seul facteur $F=31,005$, $p=0,0001)$.

La valeur de ce rapport est de $0,8 \pm 0,15$, chez les veaux âgés d'l mois, et s'élève à 2,9 $\pm 0,2(p<0,001)$ au $4^{\mathrm{e}}$ mois, puis reste relativement stable jusqu'à l'âge de 9 mois où l'urine est environ quatre fois plus concentrée que le plasma (tableau I).

\section{Activité rénine plasmatique (ARP)}

Une valeur moyenne de $0,89 \pm 0,1 \mathrm{nmol}$ d' $\mathrm{AgI} / \mathrm{L} / \mathrm{h}$ a été mesurée chez les veaux âgés d'1 mois. Cette moyenne est similaire à celle rencontrée chez les adultes; l'ARP ne varie donc pas significativement en fonction de l'âge (tableau II).

\section{Aldostérone plasmatique}

Les résultats concernant l'évolution de l'aldostéronémie en fonction de l'âge des veaux sont illustrés dans le tableau II.

Les concentrations de l'aldostérone plasmatique restent relativement stables au cours du développement post-natal.

\section{Cortisol plasmatique}

L'évolution de la cortisolémie en fonction de l'âge chez les veaux montre des valeurs légèrement élevées durant les 3 premiers mois de la vie postnatale. Toutefois, la différence observée n'est pas significative (tableau II).

\section{DISCUSSION}

Il n'est pas crucial pour les mammifères d'avoir un rein totalement opérationnel dès la naissance, étant donné que la période d'allaitement fournit aux nouveau-nés un lait maternel de composition hydrique et électrolytique relativement stable.

Les résultats obtenus chez le veau montrent que le pouvoir de concentration rénal estimé par le rapport osmolaire urine/plasma croît avec l'âge et se stabilise aux alentours $\mathrm{du} 4^{\mathrm{e}}$ mois, période durant laquelle les veaux excrètent une urine trois fois plus concentrée que le plasma. Comparativement, chez d'autres espèces, cette stabilité de l'osmolalité urinaire est atteinte à 2 mois chez le rat (Bonvalet et al, 1972 ; Trin-Tring-Tan et al, 1987) et à 77 jours chez le chien (Horster et Valtin, 1971).

La production d'une urine d'osmolalité faible durant les premiers mois de la vie post-natale chez les veaux pourrait être secondaire à un défaut de la perméabilité des tubules collecteurs qui s'installerait avec l'âge et induirait donc l'augmentation de la concentration urinaire. Cette idée, 


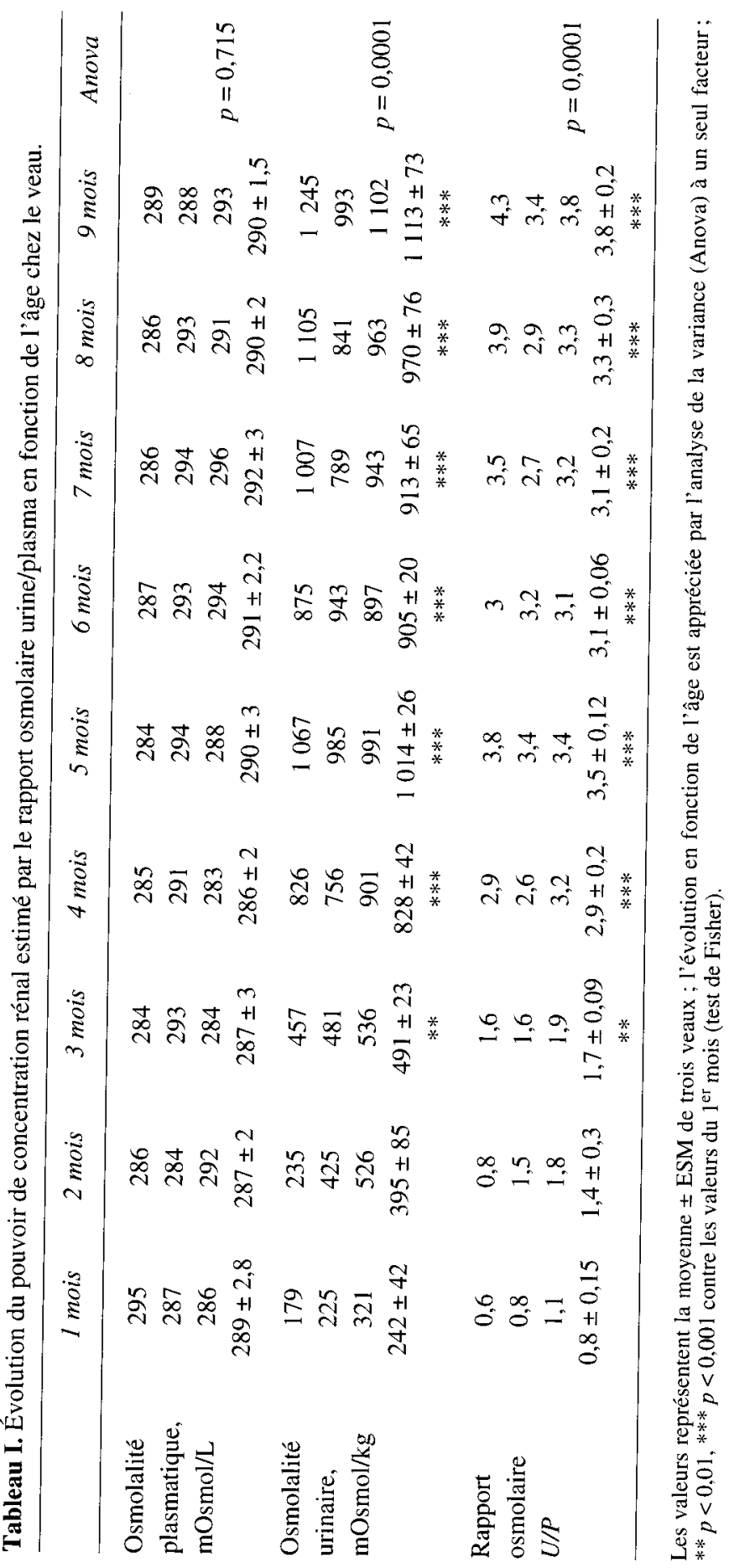




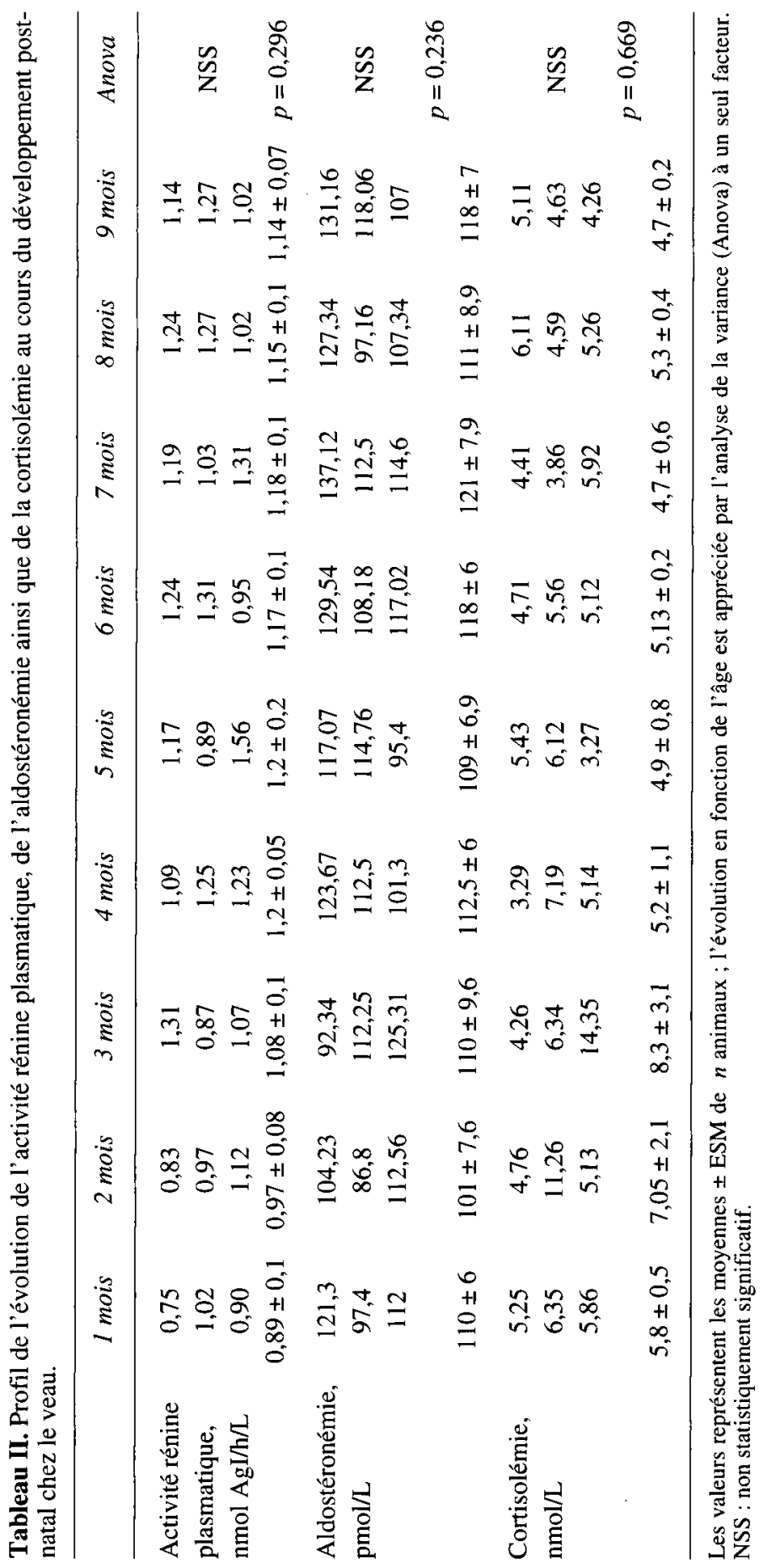


notée par Heller et Lederis (1959), a permis de penser soit à une absence d'ADH circulante, soit à une inefficacité du couplage hormone-récepteur durant les premiers jours de la vie post-natale. Toutefois, des études faites chez le rat montrent qu'une ADH immunoréactive est détectée dans l'hypothalamus à la fin de la vie foetale (Leake et Weitzman, 1979 ; Laurent et al, 1989). En outre, le rat nouveau-né est capable de répondre à une stimulation osmotique ou à une déshydratation par une augmentation du taux d'ADH circulant (Sinding et al, 1980) alors que la capacité de dilution du segment médullaire de l'anse ascendante et le pouvoir du rein à concentrer l'urine se développent seulement au moment du sevrage de l'animal qui correspond à l'âge de 3 mois chez les veaux de la race étudiée (Edwards et al, 1982; Lelièvre-Pégorier et al, 1983). De plus Rajerison et Butlen (1976) ont montré sur des fractions membranaires de la médulla rénale du rat, une augmentation avec l'âge de l'activité adénylate cyclase dépendante de l'ADH. L'ontogenèse des récepteurs de l'ADH au niveau des tubules collecteurs isolés du cortex de la médulla externe ou de la médulla interne montre que le nombre de ces récepteurs reste constant durant les premières semaines après la naissance, et n'augmente qu'après l'âge de 20 jours et atteint les niveaux retrouvés chez l'adulte 5 semaines après la naissance (Ammar et al, 1992). De plus, d'importants changements morphologiques et fonctionnels ont lieu dans le rein durant la période post-natale (Aperia et Elinder, 1981 ; Merlet-Bénichou et Rouffignac, 1977 ; Robillard et Nakamura, 1988 ; Sudelin et Bohman, 1990). Ainsi le nombre de néphrons augmente avec l'âge et, chez le rat nouveau-né, le nombre de néphrons à la naissance ne représente qu'un tiers de celui de l'adulte (Solomon, 1977), ce qui pourrait expliquer en partie que le débit de filtration glomérulaire croît linéairement avec l'âge (Horster et Valtin, 1971). Chez les jeunes veaux, l'évolution du nombre des néphrons durant la période postnatale n'est pas encore connue. Par ailleurs, il a aussi été observé (El Dahr et Chao, 1992) que les anses de Henlé, qui sont courtes à la naissance, se développent progressivement pendant les premières semaines de la vie post-natale permettant d'atteindre des structures médullaires internes de plus en plus profondes, favorisant de ce fait un important recyclage du sodium et de l'urée, phénomène à la base de l'instauration du gradient osmotique cortico-papillaire. Ainsi, chez le veau âgé de 9 mois, le rapport $U / P$ est multiplié par un facteur de 4 mettant en évidence le développement du pouvoir de concentration rénal puisque l'osmolalité urinaire augmente simultanément alors que celle du plasma ne varie pas. Cette augmentation de l'osmolalité urinaire serait la conséquence de l'augmentation de la séquestration de l'urée au niveau de la médulla rénale (Horster et Valtin, 1971).

Par contre, la régulation hormonale du métabolisme hydrominéral est relativement stable au cours du développement post-natal. Ainsi, les valeurs de l'ARP rencontrées chez les veaux âgés d' 1 mois sont dans le même ordre de grandeur que celle de l'adulte. Dans le même sens, Amadieu-Farmakis et al (1988) ont rapporté des valeurs de l'ARP chez les veaux âgés de 5 jours similaires à celles de l'adulte, ce qui permet de penser que la maturation du système rénine angiotensine s'installe relativement tôt dans la vie de l'animal. En effet, durant le développement rénal, les cellules immunoréactives contenant la rénine sont localisées chez le rat tout au long de l'arcade artérielle rénale (Mc Reddie et al, 1994). Puis, au fur et à mesure de la progression de la maturation rénale, la distribution de ces cellules myoépithéliales remonte de plus en plus et se confine à la localisation classique rencontrée chez les adultes.

Les variations de l'aldostérone dépassent rarement les premières semaines post- 
natales, puis les valeurs des concentrations circulantes deviennent égales à celles mesurées chez l'adulte (Safwate et al 1981, Safwate, 1985). Il semble que le taux de la majorité des hormones subit des modifications importantes juste après la naissance (Flairclough et al, 1975). Ainsi, la cortisolémie chute juste après la naissance, puis se maintient dans les limites des valeurs décrites chez l'adulte (Flairclough et al, 1975 ; Thomas et al, 1976).

Il apparaît donc que la maturité du développement de la fonction rénale chez le veau s'installe à l'âge de 3 mois. Cette stabilité dépend surtout de l'acquisition par le rein de toutes ses potentialités structurales qui sont d'une importance capitale dans le processus du pouvoir de concentration du rein. Bien que les concentrations des hormones étudiées soient identiques aux niveaux circulants mesurés chez l'adulte, il est possible que l'évolution en fonction de l'âge du nombre et/ou du couplage des récepteurs avec ces médiateurs hormonaux puisse expliquer l'amélioration du pouvoir de concentration du rein durant la période postnatale.

\section{RÉFÉRENCES}

Amadieu-Farmakis M, Barlet JP, Giry K, Kati-Coulibaly S (1988) Plasma atrial natriuretic peptide (ANP) and vasopressin changes in calves during the postnatal period. $J$ Physiol (Lond) 406, 131

Ammar A, Roseau S, Butlen D (1992) Postnatal ontogenesis of vasopressin receptors in the collecting duct. Mol Cell Endocrinol 86, 193-203

Aperia A, Elinder G (1981) Distal tubular sodium reabsorption in the developping rats kidney. Am J Physiol 240, 487-491

Baddouri Kh, Veillat J, Hilali M (1975) Microdissection of the proximal part of the nephron in Jaculus orientalis and Jaculus deserti. Arch Biol Nanka Beograd 27, 15-22

Baron R (1990) Anatomie comparée des mammiferes domestiques, splanchnologie. Tome 4. Éd Vigot, Paris, France

Bonvalet IP, Champion M, Wanstok F, Berjal G (1972) Compensatory renal hypertrophy in young rats: increase in the number of nephrons. Kidney Int 1 , 391-402

El-Dahr S, Chao J (1992) Spatial and temporal expression of kallikrein and its mRNA during nephron maturation. Am J Physiol 262, 705-711

Edwards BR, Mendel DB, La Rochelle FT, Stern Jr P, Valtin H (1982) Postnatal development of urinary concentrating ability in rats: changes in renal anatomy and neurohypophyseal hormones. In : The Kidney during development, Morphology, Function. (A Spitzer, New York, USA, Masson, Paris, France, 233-240

Flairclough RJ, Hunter JT, Welch RAS, Payne E (1975) Plasma corticosteroid concentration in the bovin fotus near term. I Endocrinol $65,139-140$

Heller H, Lederis K (1959) Maturation of the hypothalamo-neuro-hypophyseal system. J Physiol (Lond) 147,299

Horster M, Valtin H (1971) Postnatal development or renal function: micropuncture and clearance studies in the dog. J Clin Invest 50, 779-795

Laurent FM, Hindelang C, Stoeckel ME, Felix JM (1989) Expression of oxytocine and vasopressin genes in the rat hypothalamus during the development; an in situ hybridation study. Dev Brain Res 46, 145-155

Leake RD, Weitzman RE (1979) Developmental pharmacokinetics of the posterior pituitary hormones. Clin Pharmacol 6, 65

Lelièvre-Pégorier M, Merlet-Benichou C, Roinel N, De Rouffignac C (1983) Development pattern of water and electrolyte transport in rat superficiel nephrons. Am J Physiol 245, 15-21

Mc Reddie AT, Johns DW, Geary KM, Dagli H, Evertt AS, Chevalier RL, Carey RM, Gomez RA (1994) Angiotensin II type 1 receptor: role in renal growth and gene expression during normal development. Am J Physiol 266, 911-918

Merlet-Benichou C, Rouffignac C (1977) Renal studies in fœtal and young guinea-pigs: effect of salt loading. Am J Physiol 232, 178-185

Rajerison RM, Butlen D (1976) Ontogenic development of antiduiretic hormone receptors in rat kidney. Comparison of hormonal binding and adenylate cyclase activation. Mol Cell Endocrinol 4, 271

Robillard JE, Nakamura KT (1988) Neurohormonal regulation of renal function during development. Am J Physiol 254, 771-779

Safwate A (1985) Urinary sodium excretion and renin aldosteron system in the newborn calves. $J P h y$ siol (Lond) 362, 261-271

Safwate A, Richet E, Dalle M, Barlet JP (1981) Influence d'une perfusion d'ACTH sur l'aldosteronémie du veau. $J$ Physiol (Paris) 77, 24A 
Sinding C, Robinson AG, Seif SM (1980) Levels of neurohypophyseal peptides in the rat during the first month of life. II. Responses to physiological stimuli. Endocrinology 107, 755-763

Solomon S (1977) Developmental changes in nephron number, proximal tubular length and superficial nephron glomerular filtration rate of rats. $J P h y-$ siol 282, 573-589

Sudelin B, Bohman SO (1990) Postnatal development of the interstitial tissue of rat kidney. Anat Embryol $182,307-317$
Thomas SJ, Wilson DW, Pierre-Point CG, Ameron EHD, Griffiths K (1976) Measurement of cortisol, cortisone, 11-deoxy-cortisol and cortisone in fotal sheep plasma during the perinatal period. $J$ Endocrinol 68, 181-189

Trin-Trang-Tan MM, Bouby N, Kriz W, Bankir L (1987) Functional adaptation of think ascending limb and internephron heterogeneity to urine concentration. Kidney Int 31, 549-555

Valtin H (1977) Structural and functional heterogeneity of mammalian nephrons. Am J Physiol 233, 491-501 